\title{
Tunable spin-flop transition in artificial ferrimagnets
}

\author{
N. O. Antropov $\odot,{ }^{1,2}$ E. A. Kravtsov $\odot,{ }^{1,2}$ M. V. Makarova $\odot,{ }^{1,2}$ V. V. Proglyado, ${ }^{1}$ T. Keller $\odot,{ }^{3,4}$ I. A. Subbotin, ${ }^{5}$ \\ E. M. Pashaev, ${ }^{5}$ G. V. Prutskov, ${ }^{5}$ A. L. Vasiliev, ${ }^{5,6}$ Yu. M. Chesnokov, ${ }^{5}$ N. G. Bebenin $\odot,{ }^{1}$ \\ M. A. Milyaev, ${ }^{1}$ V. V. Ustinov $\left(1,{ }^{1}\right.$ B. Keimer, ${ }^{3}$ and Yu. N. Khaydukov ${ }^{3,4,7}$ \\ ${ }^{1}$ Institute of Metal Physics, 620108 Ekaterinburg, Russia \\ ${ }^{2}$ Ural Federal University, 620002 Ekaterinburg, Russia \\ ${ }^{3}$ Max-Planck-Institut für Festkörperforschung, Heisenbergstraße 1, D-70569 Stuttgart, Germany \\ ${ }^{4}$ Max Planck Society Outstation at the Heinz Maier-Leibnitz Zentrum (MLZ), D-85748 Garching, Germany \\ ${ }^{5}$ National Research Center “Kurchatov Institute," 123182 Moscow, Russia \\ ${ }^{6}$ Moscow Institute of Physics and Technology, 141701 Dolgoprudniy, Russia \\ ${ }^{7}$ Skobeltsyn Institute of Nuclear Physics, Moscow State University, Moscow 119991, Russia
}

(Received 18 January 2021; revised 11 June 2021; accepted 27 July 2021; published 10 August 2021)

\begin{abstract}
Spin-flop transition (SFT) consists in a jump-like reversal of antiferromagnetic (AF) lattice into a noncollinear state when the magnetic field increases above the critical value. Potentially the SFT can be utilized in many applications of a rapidly developing AF spintronics. However, the difficulty of using them in conventional antiferromagnets lies in (a) too large switching magnetic fields (b) the need for presence of a magnetic anisotropy, and (c) requirement to apply magnetic field along the correspondent anisotropy axis. In this work we propose to use artificial ferrimagnets (FEMs) in which the SFT occurs without anisotropy and the transition field can be lowered by adjusting exchange coupling in the structure. This is proved by experiment on artificial Fe-Gd FEMs where usage of Pd spacers allowed us to suppress the transition field by two orders of magnitude.
\end{abstract}

DOI: 10.1103/PhysRevB.104.054414

\section{INTRODUCTION}

Antiferromagnetic (AF) spintronic is nowadays a rapidly developing area [1-5]. In addition to nonvolatility of conventional ferromagnetic spintronics the AF devices can offer immunity to external magnetic disturbances, absence of crosstalks between small-area devices and much faster dynamics ( $\mathrm{THz}$ vs $\mathrm{MHz}$ ). The AF systems are featured by spin-flop transition (SFT) when there is the transition from AF ordering to noncollinear (NC) state at magnetic field exceeding certain value $H_{\mathrm{SP}}$. Creation of $\mathrm{NC}$ magnetic state and possibility to switch between AF and NC states may have useful applications by utilizing anomalous Hall or Nernst effects [6-11]. In addition, proximity of NC magnetic texture to superconducting layer generates long-range triplet superconductivity which may also find diverse applications in superconducting spintronics [12-16]. The utilization of the spin-flop effect in AF systems is overly complicated due to at least two reasons. The first thing is the existence of SFT in AF requires uniaxial anisotropy and an external field applied along the corresponding axis. Second, typical transition fields $H_{\mathrm{SP}}$ in bulk antiferromagnets are tens of Tesla [17-20], which is too high for real applications. The need to have anisotropy inside

Published by the American Physical Society under the terms of the Creative Commons Attribution 4.0 International license. Further distribution of this work must maintain attribution to the author(s) and the published article's title, journal citation, and DOI. Open access publication funded by the Max Planck Society. the system can be circumvented by replacing antiferromagnets with ferrimagnets (FEMs). In the FEMs one does not require presence of anisotropy and the SFT takes place at $H_{\mathrm{SP}}=\lambda\left|m_{1}-m_{2}\right|$ [21], where $m_{1,2}$ are the magnetic moment of first and second sublattices and $\lambda$ is the exchange parameter. In bulk systems the $H_{\text {SP }}$ are still too high for applications and can hardly be tuned.

In contrast, artificial FEMs based on magnetic heterostructures give a possibility to tune the SFT field by varying parameters of ferromagnetic layers and by introducing nonmagnetic spacers. Heterostructures based on $3 \mathrm{~d}$ transition metals (TM) and heavy 4f rare-earth (RE) metals, like Fe/Gd, are model FEM systems demonstrating a rich magnetic phase diagram with complex types of magnetic ordering [22-27]. Coupling between $4 \mathrm{f}$ electrons of $\mathrm{Gd}$ and $3 \mathrm{~d}$ electrons of Fe leads to the AF alignment of TM and RE magnetic moments which due to the difference in magnetic moments of $\mathrm{Fe}\left(\sim 2 \mu_{B}\right)$ and $\mathrm{Gd}\left(\sim 7 \mu_{B}\right)$ leads to the emergence of a one-dimensional FEM lattice. The SFT was found in $\mathrm{Gd} / \mathrm{Fe}$ systems at typical value $H_{\mathrm{SP}} \sim 3 \mathrm{kOe}$ [28], which is much smaller than that for bulk FEMs but still quite high for applications. Further tuning of $H_{\mathrm{SP}}$ can be gained by suppression of interlayer exchange coupling which can be performed by spacing of $\mathrm{Fe}$ and $\mathrm{Gd}$ with a nonmagnetic material like $\mathrm{Cr}$ [29,30], Pt [31], or Si [32]. The SFT can be detected by integral magnetic techniques as a kink on a magnetic hysteresis loop at $H_{\text {SP. }}$ In case of artificial FEMs magnetic signal from thin films is heavily polluted by dia- or paramagnetic signal of thick substrates. This makes it difficult, if not impossible at all, to use integral magnetometric methods to study the SFTs. 
Neutron scattering, being a depth-selective magnetometric technique is a widely used method for studying AFs and FEMs [33-35]. Similar to X-ray and light, neutrons diffract at periodic lattice with period $D$ according to the well-known Bragg law $n \lambda=2 D \sin \theta$. Here $\lambda$ and $\theta$ are the neutron wavelength and incident angle, and $n$ is integer number corresponding to order of Bragg peak. Presence of spin one-half makes neutron scattering sensitive to the magnetic lattice. In case of AF magnetic lattice is doubled comparing to the structural one, so that the magnetic Bragg peak appears on the positions of $n / 2$ of the structural Bragg peaks. Applying spin analysis, that is detecting neutron spin-states before and after scattering, allows one to get additional information about magnetic configuration. The nonspin-flip (NSF) channels $(++)$ and $(--)$ are sensitive to the sum and difference of nuclear potential and collinear to the neutron polarization part of magnetization. Here first and second sign codes neutron polarization along the external magnetic field $H$ before and after the scattering process. Presence of noncollinear magnetization causes spin-flip (SF) scattering $(+-)$ and $(-+)$. In Born approximation the amplitude of the SF scattering is proportional to the spatial profile of the NC magnetization in reciprocal space. Thus, the measurement of the SF scattering is a direct method for detecting of SFT.

In our prior work [36] we studied superlattice $[\mathrm{Fe}(3.5 \mathrm{~nm}) / \operatorname{Pd}(1.2 \mathrm{~nm}) / \operatorname{Gd}(5 \mathrm{~nm}) / \operatorname{Pd}(1.2 \mathrm{~nm})]_{12}$. In the neutron experiment we measured intensity of the SF scattering at the position of the first Bragg peak $R_{1}^{\mathrm{SF}}$ as a function of external magnetic field at a temperature of $10 \mathrm{~K}$. Above magnetic field of $\mathrm{H}_{S P}=1.5 \mathrm{kOe}$ we detected a 20 -fold increase of SF scattering which is the direct evidence for the presence of SFT in our system. We note that the $H_{\mathrm{SP}}$ field is much smaller than in spacer free Fe/Gd systems. Subsequent structural studies by transmission electron microscopy, and X-ray reflectometry (XRR) [37] indicated presence of mutual diffusion at $\mathrm{Gd} / \mathrm{Pd}$ interface. For thin $(\sim 1 \mathrm{~nm})$ Pd spacers this interdiffusion leads to almost complete dissolution of $\mathrm{Pd}$ in $\mathrm{Gd}$. As a result the Curie temperature $T_{m}$ (and hence exchange energy) of the (nominal) Gd layer decreases from $T_{m}=294 \mathrm{~K}$ for bulk Gd to $T_{m} \lesssim 100 \mathrm{~K}$. Thus ability of Pd and Gd to form an alloy with controllable suppression of exchange energy paves the way for tuning of SFT by varying thickness of Pd spacer, which we aim to study in this work.

\section{SAMPLE FABRICATION AND EXPERIMENTAL TECHNIQUES}

Using an UHV magnetron machine ULVAC MPS-4000C6 we prepared series of samples of nominal composition $[\mathrm{Fe}(35 \AA) / \operatorname{Pd}(t) / \operatorname{Gd}(50 \AA) / \operatorname{Pd}(t)]_{12}$ with $t=8 \AA, 10 \AA$, $12 \AA, 14 \AA, 16 \AA$, and $28 \AA$. Further we will code samples as PdYY, where YY is thickness of Pd layer in Angstroms. The growth of the films was performed at room temperature at typical rates of $25 \AA / \mathrm{min}, 55 \AA / \mathrm{min}, 80 \AA / \mathrm{min}$ for Fe, Gd, and Pd layers, correspondingly. The first deposited in series sample Pd12 had the thickness of the iron and gadolinium layers $\sim 5 \%$ less than the nominal values. For subsequent samples the deposition time of Fe and Gd layers was corrected.

The structural quality of the superlattices was attested using the $\mathrm{x}$-ray low-angle diffraction (reflectometry, XRR) with
Cu K- $\alpha$ radiation $(\lambda=1.54 \AA$ ). Additionally we performed scanning transmission electron microscopy (STEM) with the energy dispersive $\mathrm{X}$-ray (EDX) microanalysis [37].

The SQUID magnetometer Quantum Design MPMS 3 was used to measure temperature and field dependencies of the magnetic moment. For the comparative analysis of the different samples we normalized magnetic moments on area of substrate. Polarized neutron reflectometry (PNR) experiment was conducted on the monochromatic $(\lambda=4.3 \AA)$ reflectometer NREX of the research reactor FRM-2 (Garching, Germany). The polarized neutron beam (with polarization $99.99 \%$ ) was incident on a sample at grazing angles $\theta=$ $\left(0.15-6^{\circ}\right)$. The polarization of the reflected beam was analyzed by an analyzer with efficiency $99.3 \%$. In order to draw the $H-T$ phase diagram of the SFT, we measured the temperature dependencies of $R_{1}^{\mathrm{SF}}$ using following protocol. The sample was cooled to $T=5 \mathrm{~K}$ in a magnetic field of $H=1$ kOe. After that, the $H$-dependence of $(+-)$ channel was measured by increasing the magnetic field from $H_{\min }=10$ Oe to $H_{\max }=4.5 \mathrm{kOe}$ with a step of $\Delta H=150$ Oe. After that, the temperature increased by $5-10 \mathrm{~K}$ and the field dependence was measured by decreasing the magnetic field from $H_{\max }$ to $H_{\text {min }}$. With a subsequent increase in temperature, the field dependence was measured again by increasing the field, and so on. Such procedure allowed us to measure the phase diagram for one sample with 200-300 points within 3-5 h. Magnetic field in all SQUID and PNR experiments was applied parallel to sample surface.

\section{STRUCTURAL PROPERTIES}

The XRR curves measured on the samples under the study are shown in Fig. 1(a). For all samples we measured $n>10$ Bragg peaks proving thus high quality of the superlattices in terms of high repeatability of a $\mathrm{Fe} / \mathrm{Pd} / \mathrm{Gd} / \mathrm{Pd}$ unit cell [38]. Figure 1(b) shows the EDX microanalysis of STEM of Pd12 sample. The EDX analysis shows well-defined Fe layers depicted by the blue color. However, instead of separate Gd (red) layers and Pd (green) spacers one can see mixed layers of GdPd (yellow) alloy through the whole structure, except the first and the last layers. Here one can still observe well-defined layers of pure Gd. For the sake of simplicity, we will keep naming Gd layer, remembering, however, that in reality the

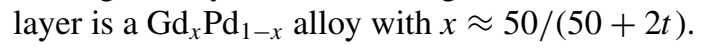

\section{MAGNETIC PROPERTIES}

Figure 2(a) shows the hysteresis loops measured on samples $\mathrm{Pd} 28$ and $\mathrm{Pd} 10$ at a temperature of $5 \mathrm{~K}$. The loop for Pd10 sample has a typical for FEMs form with plateau in the AF state followed by a kink at the SFT with $H_{\mathrm{SP}}=1.6$ kOe. This kink was absent for all samples with $t>14 \AA$ [inset in Fig. 2(a)]. Figure 2(b) shows the temperature dependence of magnetic moment measured in a magnetic field of $H=$ $4.5 \mathrm{kOe}$. At $T>T_{m}$ the curves change rather weakly compared with the room temperature moment. The slight change is most likely due to layers of undiluted gadolinium at the edges of the sample. At $T<T_{m}$ an increase in the magnetic moment associated with the transition of the GdPd layer to the 
(a)

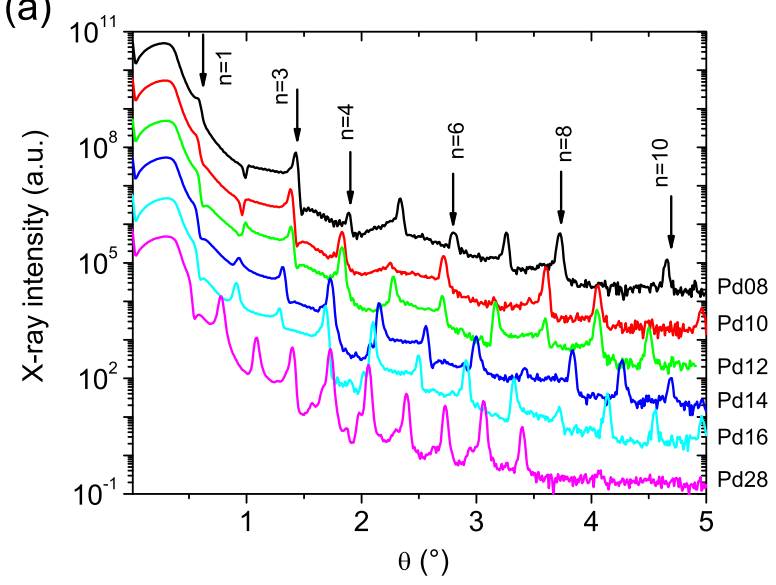

(b)

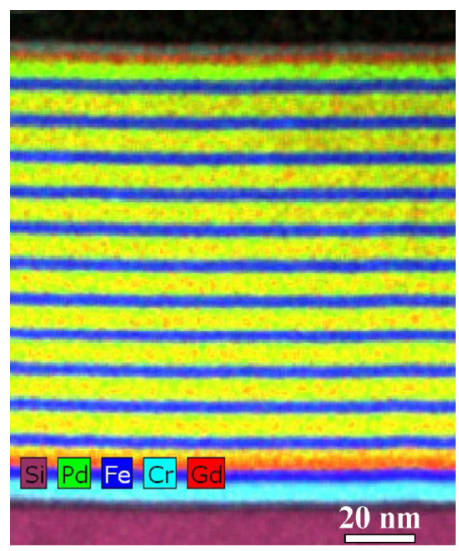

FIG. 1. (a) X-ray low-angle diffraction (reflectivity) of samples under study. Vertical arrows show the position of several Bragg peaks for sample Pd08. (b) The energy dispersive X-ray (EDX) microanalysis of Pd12 sample.

ferromagnetic state is observed. The transition point $T_{m}$ shifts to lower temperatures with increasing $t$ [inset in Fig. 2(b)].

Figure 3 shows the PNR data measured on sample Pd10 at $T=10 \mathrm{~K}$ in magnetic field $H=1 \mathrm{kOe}$ and additional SF curve at $T=10 \mathrm{~K}$ in magnetic field $H=3 \mathrm{kOe}$ (solid line). In the neutron experiment, 4 Bragg peaks were confidently measured. A large splitting of (++) and (--) NSF Bragg peaks indicates the presence of a collinear magnetic moment in the system. At the same time we observed a much weaker (1-2 orders below NSF signal) SF scattering at Bragg peaks. The origin of this small, though not negligible, SF signal can be associated with $\mathrm{NC}$ inhomogeneities at the Fe/Gd interfaces. The data at $H=1$ kOe can be quantitatively described by a predominantly collinear AF state with magnetic moments of Gd $M_{\mathrm{Gd}} \approx 5 \mu_{B}$ and $\mathrm{Fe} M_{\mathrm{Fe}} \approx 2 \mu_{B}$ aligned parallel and antiparallel to $H$. By increasing the magnetic field above $H_{\mathrm{SP}}=$ $2.3 \mathrm{kOe}$ (inset in Fig. 3), we observed a 20 -fold increase of $\mathrm{SF}$ scattering at the first Bragg peak $R_{1}^{\mathrm{SF}}$. We note that the field $H_{\mathrm{SP}}$ at $10 \mathrm{~K}$ measured by PNR is somewhat larger than the field at $5 \mathrm{~K}$ from SQUID and PNR, which is apparently associated with the hysteresis behavior [see Fig. 2(a) and inset].

By measuring the family of $R_{1}^{\mathrm{SF}}(\mathrm{H})$ scans at different temperatures we were able to construct the $\mathrm{NC}$ magnetic phase diagram for the sample Pd10 in $H-T$ coordinates [Fig. 4(a)]. For this sample we observe a collinear AF state in the temperature range up to $30 \mathrm{~K}$ in magnetic fields not exceeding $2 \mathrm{kOe}$. Above this field, the collinear AF state is replaced by a NC spin-flop state. Increasing the temperature to $60 \mathrm{~K}$ leads to a gradual shift of the SFT field toward lower values. Finally, above $60 \mathrm{~K}$, the spin-flip signal disappears due to the absence of magnetic ordering in $\mathrm{Gd}$ layer. Figures 4(b) and 4(c) shows similar phase diagrams for Pd12 and Pd14 samples. One can see that the transition field $H_{\mathrm{SP}}$ decreases with increase of $t$. For the samples with $t=1.6 \mathrm{~nm}$ (not shown) we did not observe any detectable

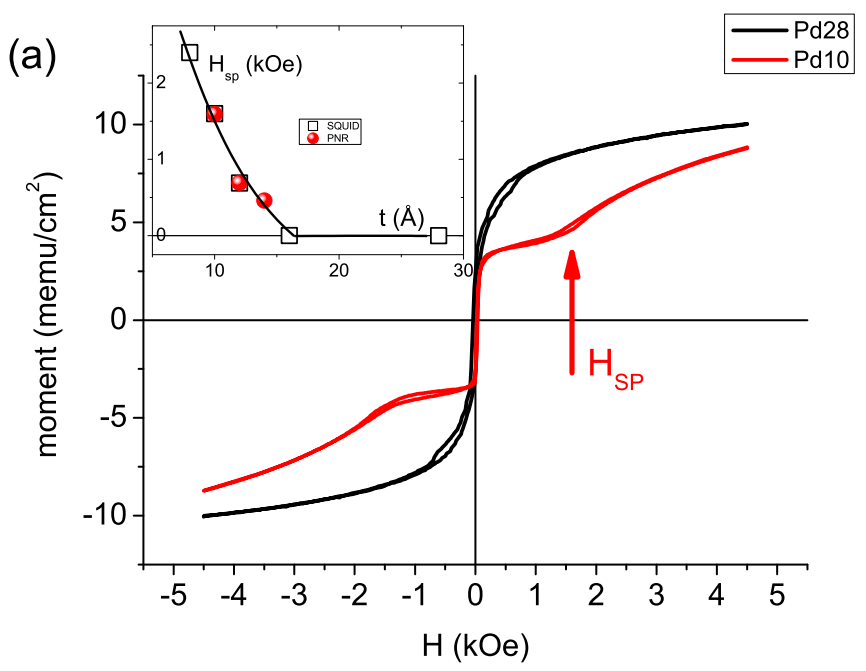

(b)

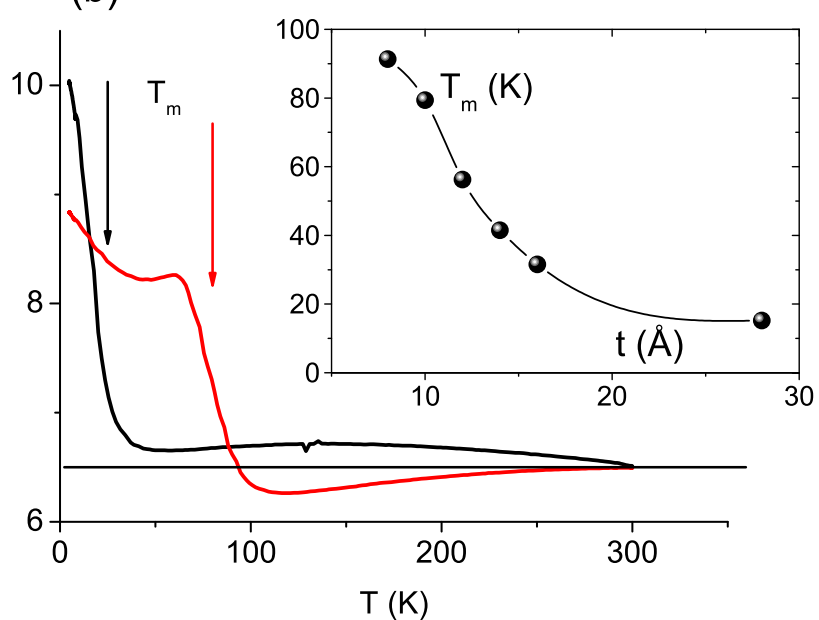

FIG. 2. The field dependence (a) at $T=5 \mathrm{~K}$ and temperature dependence (b) at $H=4.5 \mathrm{kOe}$ of the magnetic moment of samples Pd10 and Pd28. The vertical arrow in (a) shows the position of $H_{\mathrm{SP}}$ for the sample Pd10. Inset in (a) shows the $t$ dependence of $H_{\mathrm{SP}}$ from SQUID (open dots) and PNR (solid circles). The line in the inset is to guide the eye. Vertical black and red arrows in (b) show the transition temperature $T_{m}$ for samples Pd28 and Pd10, respectively. Inset in (b) shows the $t$ dependence of $T_{m}$. 


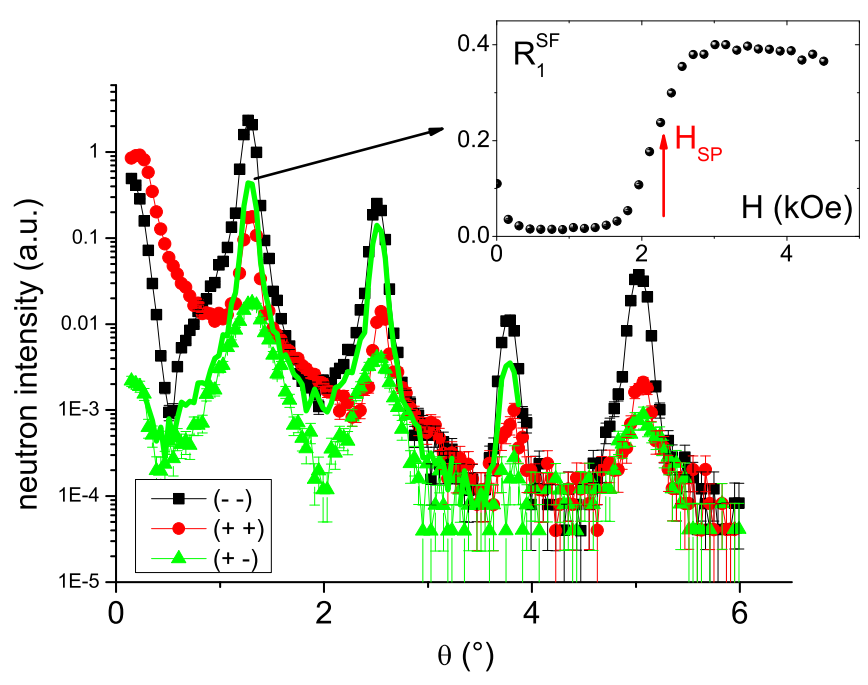

FIG. 3. Polarized neutron reflectivities of sample Pd10 measured at $T=10 \mathrm{~K}$ at magnetic field $H=1 \mathrm{kOe}$ (symbols) and SF curve at $T=10 \mathrm{~K}, H=3 \mathrm{kOe}$ (solid line). Inset shows the field dependence of intensity of SF scattering at the first Bragg peak $R_{1}^{\mathrm{SF}}(H)$. Vertical arrow denotes the magnetic field at which SFT takes place.

SF signal evidencing absence of coupling between Fe and $\mathrm{Gd}$ layers.

To describe the magnetic state of our systems we applied the extended Stoner-Wohlfarth model widely used for description of magnetic multilayers [8,39]. Density of magnetic energy of one $\mathrm{Fe} / \mathrm{Gd}$ unit cell can be written as

$$
\begin{aligned}
E\left(\alpha_{\mathrm{Gd}}, \alpha_{\mathrm{Fe}}\right)= & -H\left[m_{\mathrm{Gd}} \cos \left(\alpha_{\mathrm{Gd}}\right)+m_{\mathrm{Fe}} \cos \left(\alpha_{\mathrm{Fe}}\right)\right] \\
& +J_{1} \cos \left(\alpha_{\mathrm{Gd}}-\alpha_{\mathrm{Fe}}\right)+J_{2} \cos ^{2}\left(\alpha_{\mathrm{Gd}}-\alpha_{\mathrm{Fe}}\right) .
\end{aligned}
$$

In Eq. (1) $m_{X}=M_{X} d_{X}$ is a product of magnetization and thickness (magnetic moment), $\alpha_{X}$ is the angle between magnetization, and $H$ of a layer $X(X=\mathrm{Fe}, \mathrm{Gd})$. The first term in Eq. (1) is Zeeman energy, which tends to align magnetic moments of the layers along the external field. The second term is bilinear AF exchange coupling of $\mathrm{Fe}$ and $\mathrm{Gd}$ layers with strength parameter $J_{1}$. The third term describes biquadratic coupling tending to align the magnetic moments noncollinearly. As seen from Eq . (1) in case $J_{2}=0$, the transition field can be estimated as

$$
H_{\mathrm{SP}} \approx J_{1}\left|m_{\mathrm{Gd}}-m_{\mathrm{Fe}}\right| / m_{\mathrm{Gd}} \cdot m_{\mathrm{Fe}} .
$$

For every magnetic field $H$ the magnetic configuration of the system as a function of $J_{1,2}$ can be obtained by minimizing energy (1) varying angles $\alpha_{\mathrm{Gd}}$ and $\alpha_{\mathrm{Fe}}$. The magnetic moments $m_{X}$ were taken from SQUID data as follows. Due to the high Curie temperature of iron $(\sim 1000 \mathrm{~K})$, it can be assumed that the magnetic moment of iron $m_{\mathrm{Fe}}$ does not change in the studied temperature range. Hence the moment can be taken from the room temperature data where the Gd layers are not magnetic. The AF plateau at $T=5 \mathrm{~K}$ gives us differential moment $m_{\mathrm{Gd}}-m_{\mathrm{Fe}}$, allowing us to estimate $m_{\mathrm{Gd}} \approx 10(1) \mathrm{memu} / \mathrm{cm}^{2}$. We note that for 110 sample the Fe and Gd magnetic moments obtained from SQUID and PNR data agree well within the experimental accuracy.
The angles $\alpha_{\mathrm{Gd}}^{\prime}$ and $\alpha_{\mathrm{Fe}}^{\prime}$ corresponding to the minimum of energy for a given set of $H$ and $J_{1,2}$ are used to construct a theoretical SF reflectivity at the first Bragg peak in Born approximation:

$R_{1, t h}^{\mathrm{SF}}=c\left[m_{\mathrm{Gd}, \perp}^{2}+m_{\mathrm{Fe}, \perp}^{2}+2 m_{\mathrm{Gd}, \perp} m_{\mathrm{Fe}, \perp} \cos \frac{d_{\mathrm{Fe}}}{d_{\mathrm{Fe}}+d_{\mathrm{Gd}}}\right]+R_{b g}$,

where $m_{\mathrm{Gd}(\mathrm{Fe}), \perp}=m_{\mathrm{Gd}(\mathrm{Fe})} \sin \alpha_{\mathrm{Gd}(\mathrm{Fe})}^{\prime}$ is the noncollinear component of the magnetic moment of $\mathrm{Gd}(\mathrm{Fe})$ layer, $c$ is scaling constant, and $R_{b g}$ is background intensity. The latter two values were adjusted manually before the fit. We then fitted theoretical $R_{1, t h}^{\mathrm{SF}}$ to the experimental $H$-dependencies $R_{1}^{\mathrm{SF}}$ by varying $J_{1}$ and $J_{2}$. The procedure was repeated for every $T$ so that for every sample we obtained temperature dependencies of $J_{1,2}$. Figure 4(d) shows results of such a fit for sample Pd10. It is rather noticeable that despite the simplicity of the Stoner-Wohlfarth approach it allows to reproduce experimental features quite well. Figure 5(a) shows the fit-resulted $T$ dependence of the exchange energies $J_{1}$ and $J_{2}$ for the Pd10 sample. It can be seen that the bilinear term has a predominant contribution, which gradually decreases approaching the $T_{m}$. Thus our analysis showed that for a qualitative description of the SFT, a bilinear term is sufficient, but quantitatively the data are described better by including an additional biquadratic term.

The data for other samples were fitted in a similar way. Figure 5(b) shows the dependency of coupling energies on thickness of Pd spacer. As follows from the figure that the bilinear energy decreases almost linearly from $1.5 \mathrm{erg} / \mathrm{cm}^{2}$ at $t=1 \mathrm{~nm}$ to 0 at $t=1.6 \mathrm{~nm}$. Biquadratic energy in turn increases with $t$. The obtained values are of the same orders as $J_{1} \sim 0.8 \mathrm{erg} / \mathrm{cm}^{2}$ and $J_{2} \sim 0.2 \mathrm{erg} / \mathrm{cm}^{2}$ obtained in Ref. [40] for $\mathrm{Gd} / \mathrm{Pt} / \mathrm{Co}$ multilayers at $T=10 \mathrm{~K}$.

\section{DISCUSSION AND CONCLUSION}

In the present work, it is shown that the insertion of a thin $(t=10-14 \AA)$ Pd spacer between $\mathrm{Fe}(35 \AA)$ and $\mathrm{Gd}(50 \AA)$ layers allows a significant (1-2 orders of magnitude) decrease in the field of SFT. At the same time, the study of the structure indicates that in the specified range of thicknesses, Gd and $\mathrm{Pd}$ are intermixed into a homogeneous alloy with palladium concentrations varying from $29 \%$ for $t=10 \AA$ to $36 \%$ for $t=14 \AA$. This alloying led to $\sim 30 \%$ decrease of the magnetic moment of the GdPd layer. The Curie temperature of the GdPd alloy was found to decrease with an increase in the palladium concentration. The theoretical calculation performed in the mean-field approximation indicates that the amplitude of the linear exchange interaction $J_{1}$ of the GdPd layer also decreases with increasing of Pd concentration, which makes it possible to adjust the transition field [see Eq. (2)]. At the same time, the growth of the biquadratic component $J_{2}$ may indicate an increase in structural inhomogeneities, which is consistent with the conclusions of the work [37].

Our study opens perspectives for a purposeful design of artificial FEMs with an adjustable field of SFT. Thus, the FEM systems with low Curie temperature components studied in this work can be used in superconducting spintronics 

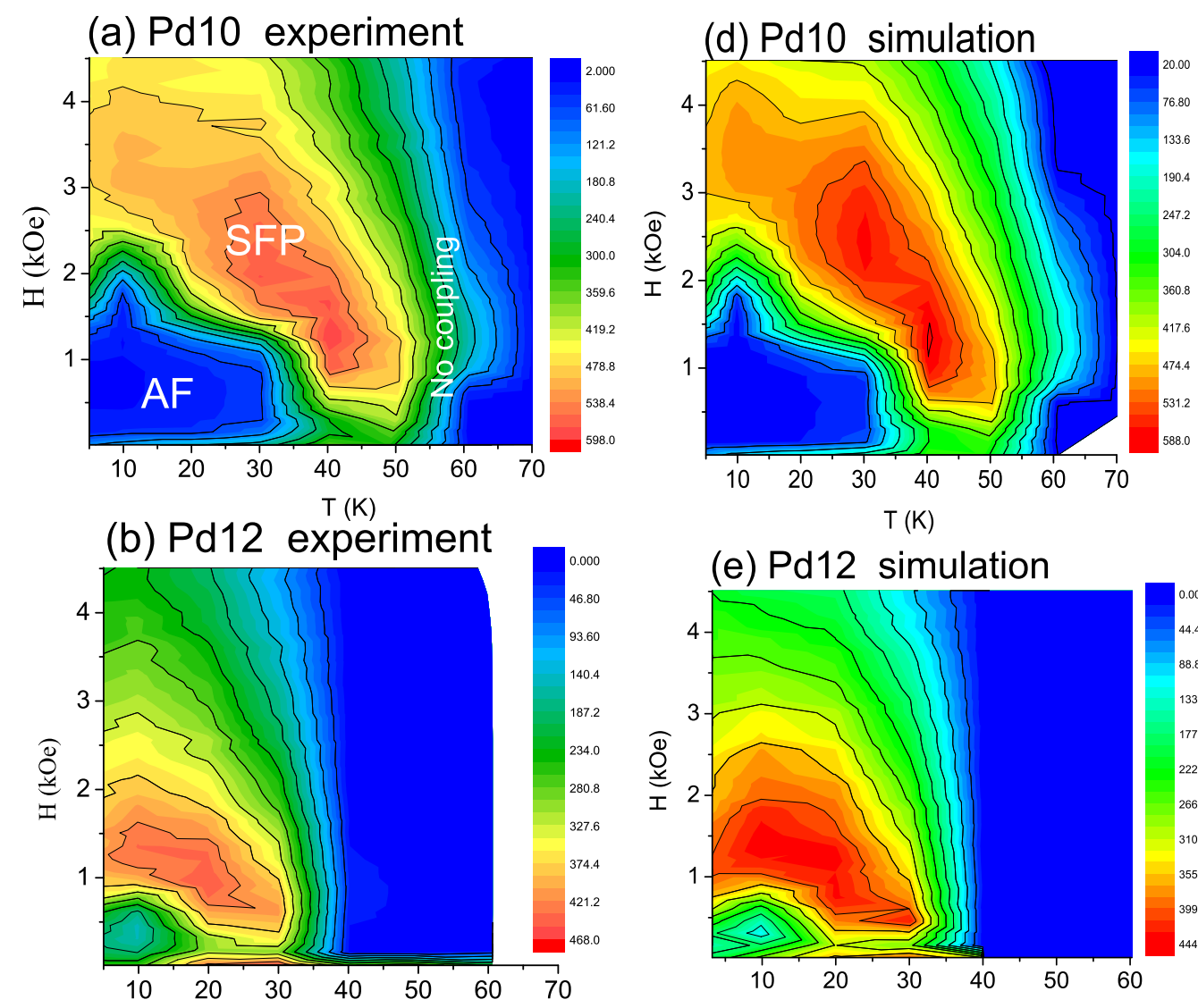

\section{(e) Pd12 simulation}

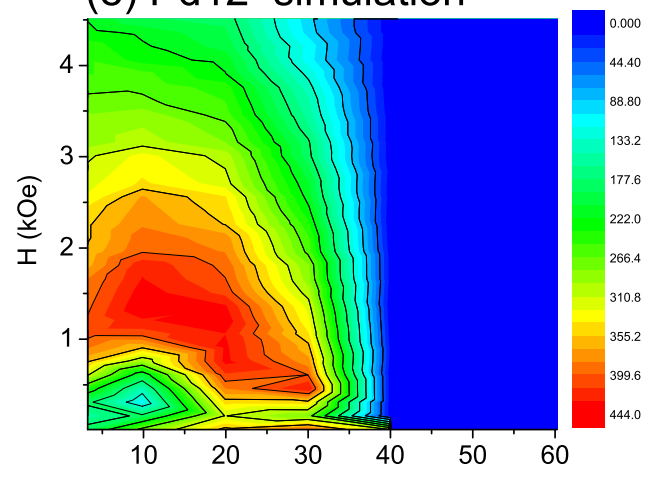

\section{(c) Pd14 experiment}

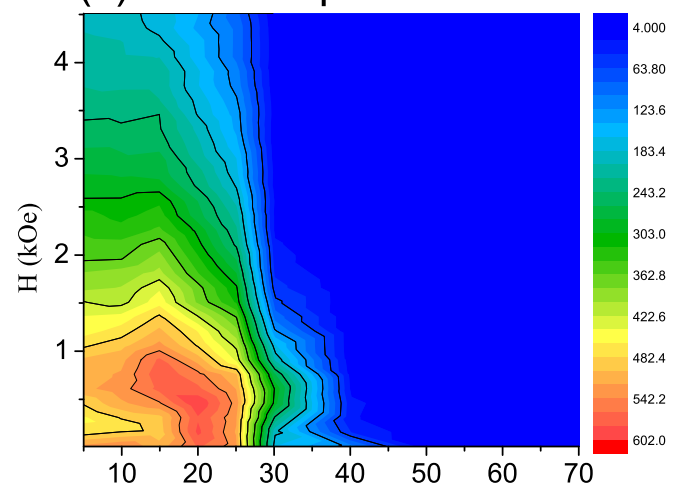

\section{(f) $\mathrm{Pd} 14$ simulation}

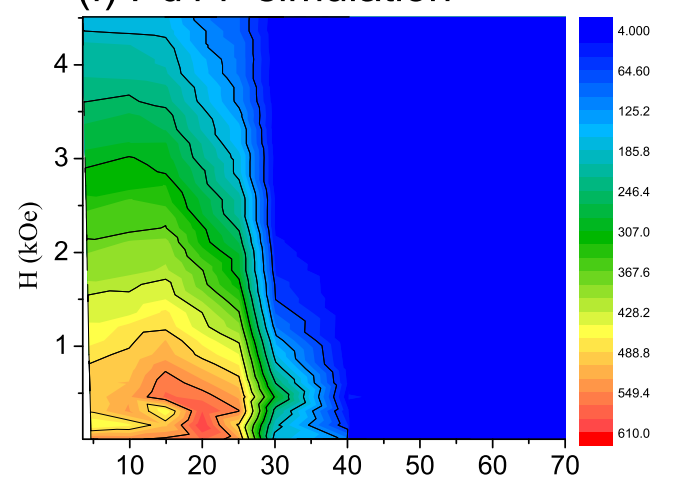

FIG. 4. (a)-(c) Experimental $(H, T)$ maps of $R_{1}^{\mathrm{SF}}$ for samples with different Pd spacers. (d)-(f) Simulated maps for these samples.

for generation of triplet superconductivitiy. In Ref. [15] it was demonstrated that a $\mathrm{Co} / \mathrm{Ru} / \mathrm{Co}$ artificial FEM structure placed between two superconducting $\mathrm{Nb}$ layers allows one to change the critical supercurrent in the structure by an order of magnitude. Advantages of our FEMs would be good compatibility of gadolinium with superconducting niobium [41,42] and reduced ferromagnetic energy, which leads to a smaller pair-breaking effect. For the room temperature applications one can use well-studied synthetic AFs such as Fe/Cr [33-35], $\mathrm{Fe} / \mathrm{V}[43,44]$, or $\mathrm{Co} / \mathrm{Cu}[45,46]$ where subsequent adjustment can be carried out by tuning of the coupling energy and the imbalance of the magnetic moments of the sublattices.
In conclusion, using PNR and complementary techniques we performed a systematic study of magnetic configuration of $[\operatorname{Fe}(3.5 \mathrm{~nm}) / \operatorname{Pd}(t) / \operatorname{Gd}(5.0 \mathrm{~nm}) / \operatorname{Pd}(t)]_{12}$ heterostructures with $t=8-28 \AA$ A. By measuring neutron spin-flip scattering we have detected the presence of a magnetically noncollinear state at temperatures $T \lesssim 50 \mathrm{~K}$ in magnetic fields of above $H>500$ Oe for the samples with $10 \AA \leqslant t \leqslant 14 \AA$. By using an extended Stoner-Wohlfarth model we were able to describe the observed transition as a competition of Zeeman energy, bilinear interaction of order of $1 \mathrm{erg} / \mathrm{cm}^{2}$, and biquadratic addition of the order of $0.5 \mathrm{erg} / \mathrm{cm}^{2}$. The coupling energies can be tuned by varying the thickness of the spacer between 
(a)

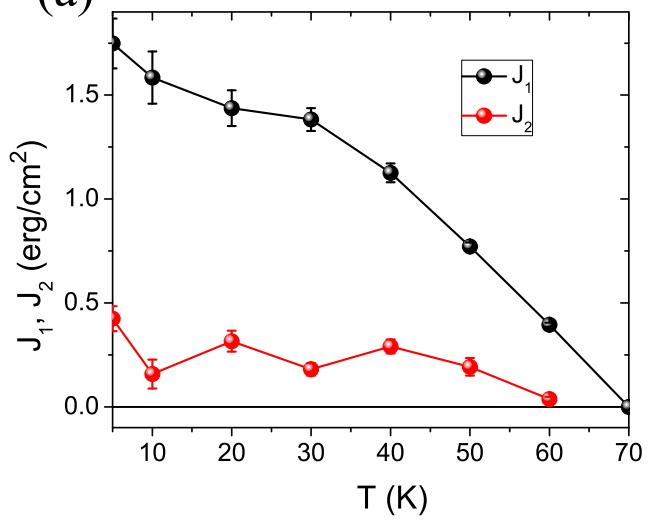

(b)

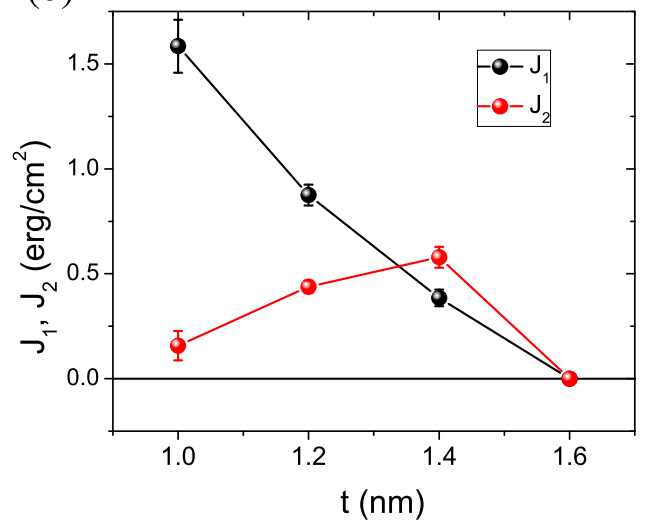

FIG. 5. (a) Fit-resulted $J_{1}$ and $J_{2}$ terms vs temperature for Pd10 sample. (b) Thickness dependence of bilinear and biquadratic energies $J_{1}$ and $J_{2}$ obtained for $T=10 \mathrm{~K}$.

1 and $1.4 \mathrm{~nm}$ leading to the shift of the transition field below kilo-Oersted range.

\section{ACKNOWLEDGMENTS}

We thank A. B. Drovosekov, D. I. Kholin, and D. Cortie for fruitful discussion of the results. This work is partially based on experiments performed at the NREX instrument operated by the Max Planck Society at the MLZ, Garching, Germany and supported by the Deutsche Forschungsgemeinschaft (Project No. 107745057-TRR80). Research in Ekaterinburg was performed within the state assignment of Minobrnauki of Russia (theme "Spin" No. AAAA-A18-118020290104-2) and was partly supported by Russian Foundation for Basic Research (Project No. 19-02-00674).
[1] A. H. MacDonald and M. Tsoi, Antiferromagnetic metal spintronics, Philos. Trans. R. Soc. A 369, 3098 (2011).

[2] T. Jungwirth, J. Sinova, A. Manchon, X. Marti, J. Wunderlich, and C. Felser, The multiple directions of antiferromagnetic spintronics, Nat. Phys. 14, 200 (2018).

[3] R. A. Duine, K.-J. Lee, S. S. P. Parkin, and M. D. Stiles, Synthetic antiferromagnetic spintronics, Nat. Phys. 14, 217 (2018).

[4] A. Hirohata, K. Yamada, Y. Nakatani, I.-L. Prejbeanu, B. Diény, P. Pirro, and B. Hillebrands, Review on spintronics: Principles and device applications, J. Magn. Magn. Mater. 509, 166711 (2020).

[5] J. Shi, V. Lopez-Dominguez, F. Garesci, C. Wang, H. Almasi, M. Grayson, G. Finocchio, and P. K. Amiri, Electrical manipulation of the magnetic order in antiferromagnetic PtMn pillars, Nat. Electron. 3, 92 (2020).

[6] H. Chen, Q. Niu, and A. H. MacDonald, Anomalous Hall Effect Arising from Noncollinear Antiferromagnetism, Phys. Rev. Lett. 112, 017205 (2014).

[7] S. Nakatsuji, N. Kiyohara, and T. Higo, Large anomalous hall effect in a non-collinear antiferromagnet at room temperature, Nature (London) 527, 212 (2015).

[8] J. D. Hoffman, B. J. Kirby, J. Kwon, G. Fabbris, D. Meyers, J. W. Freeland, I. Martin, O. G. Heinonen, P. Steadman, H. Zhou, C. M. Schlepütz, M. P. M. Dean, S. G. E. te Velthuis, J.-M. Zuo, and A. Bhattacharya, Oscillatory Noncollinear Magnetism Induced by Interfacial Charge Transfer in Superlattices Composed of Metallic Oxides, Phys. Rev. X 6, 041038 (2016).

[9] J. D. Hoffman, S. M. Wu, B. J. Kirby, and A. Bhattacharya, Tunable Noncollinear Antiferromagnetic Resistive Memory Through Oxide Superlattice Design, Phys. Rev. Applied 9, 044041 (2018).
[10] P.-X. Qin, H. Yan, X.-N. Wang, Z.-X. Feng, H.-X. Guo, X.-R. Zhou, H.-J. Wu, X. Zhang, Z.-G.-G. Leng, H.-Y. Chen, and Z.Q. Liu, Noncollinear spintronics and electric-field control: A review, Rare Metals 39, 95 (2019).

[11] S.-H. Yang, Spintronics on chiral objects, Appl. Phys. Lett. 116, 120502 (2020).

[12] F. S. Bergeret, A. F. Volkov, and K. B. Efetov, Long-Range Proximity Effects in Superconductor-Ferromagnet Structures, Phys. Rev. Lett. 86, 4096 (2001).

[13] A. F. Volkov, F. S. Bergeret, and K. B. Efetov, Odd Triplet Superconductivity in Superconductor-Ferromagnet Multilayered Structures, Phys. Rev. Lett. 90, 117006 (2003).

[14] M. Eschrig, Spin-polarized supercurrents for spintronics, Phys. Today 64(1), 43 (2011).

[15] C. Klose, T. S. Khaire, Y. Wang, W. P. Pratt, N. O. Birge, B. J. McMorran, T. P. Ginley, J. A. Borchers, B. J. Kirby, B. B. Maranville, and J. Unguris, Optimization of Spin-Triplet Supercurrent in Ferromagnetic Josephson Junctions, Phys. Rev. Lett. 108, 127002 (2012).

[16] D. Lenk, R. Morari, V. I. Zdravkov, A. Ullrich, Y. Khaydukov, G. Obermeier, C. Müller, A. S. Sidorenko, H.-A. Krug von Nidda, S. Horn, L. R. Tagirov, and R. Tidecks, Fullswitching FsF-type superconducting spin-triplet magnetic random access memory element, Phys. Rev. B 96, 184521 (2017).

[17] M. O. Yokosuk, A. al Wahish, S. Artyukhin, K. R. O'Neal, D. Mazumdar, P. Chen, J. Yang, Y. S. Oh, S. A. McGill, K. Haule, S.-W. Cheong, D. Vanderbilt, and J. L. Musfeldt, Magnetoelectric Coupling through the Spin Flop Transition in $\mathrm{Ni}_{3} \mathrm{TeO}_{6}$, Phys. Rev. Lett. 117, 147402 (2016). 
[18] F. L. A. Machado, P. R. T. Ribeiro, J. Holanda, R. L. RodríguezSuárez, A. Azevedo, and S. M. Rezende, Spin-flop transition in the easy-plane antiferromagnet nickel oxide, Phys. Rev. B 95, 104418 (2017).

[19] J. Becker, A. Tsukamoto, A. Kirilyuk, J. C. Maan, T. Rasing, P. C. M. Christianen, and A. V. Kimel, Ultrafast Magnetism of a Ferrimagnet Across the Spin-Flop Transition in High Magnetic Fields, Phys. Rev. Lett. 118, 117203 (2017).

[20] A. M. Vibhakar, D. D. Khalyavin, P. Manuel, L. Zhang, K. Yamaura, P. G. Radaelli, A. A. Belik, and R. D. Johnson, Magnetic structure and spin-flop transition in the $a$-site columnar-ordered quadruple perovskite $\mathrm{TmMn}_{3} \mathrm{O}_{6}$, Phys. Rev. B 99, 104424 (2019).

[21] A. E. Clark and E. Callen, Neel ferrimagnets in large magnetic fields, J. Appl. Phys. 39, 5972 (1968).

[22] N. Ishimatsu, H. Hashizume, S. Hamada, N. Hosoito, C. S. Nelson, C. T. Venkataraman, G. Srajer, and J. C. Lang, Magnetic structure of $\mathrm{Fe} / \mathrm{Gd}$ multilayers determined by resonant X-ray magnetic scattering, Phys. Rev. B 60, 9596 (1999).

[23] D. Haskel, G. Srajer, J. C. Lang, J. Pollmann, C. S. Nelson, J. S. Jiang, and S. D. Bader, Enhanced interfacial magnetic coupling of Gd /Fe multilayers, Phys. Rev. Lett. 87, 207201 (2001).

[24] S. A. Montoya, S. Couture, J. J. Chess, J. C. T. Lee, N. Kent, D. Henze, S. K. Sinha, M.-Y. Im, S. D. Kevan, P. Fischer, B. J. McMorran, V. Lomakin, S. Roy, and E. E. Fullerton, Tailoring magnetic energies to form dipole skyrmions and skyrmion lattices, Phys. Rev. B 95, 024415 (2017).

[25] S. A. Montoya, S. Couture, J. J. Chess, J. C. T. Lee, N. Kent, M.-Y. Im, S. D. Kevan, P. Fischer, B. J. McMorran, S. Roy, V. Lomakin, and E. E. Fullerton, Resonant properties of dipole skyrmions in amorphous Fe/Gd multilayers, Phys. Rev. B 95, 224405 (2017).

[26] K. Takanashi, Y. Kamiguchi, H. Fujimori, and M. Motokawa, Magnetization and magnetoresistance of Fe/Gd ferrimagnetic multilayer films, J. Phys. Soc. Japan 61, 3721 (1992).

[27] L. T. Baczewski, R. R. Kalinowski, and A. Wawro, Magnetization and anisotropy in Fe/Gd multilayers, J. Magn. Magn. Mater. 177, 1305 (1998).

[28] Y. Kamiguchi, Y. Hayakawa, and H. Fujimori, Anomalous field dependence of magnetoresistance in Fe/Gd multilayered ferrimagnets, Appl. Phys. Lett. 55, 1918 (1989).

[29] A. B. Drovosekov, N. M. Kreines, A. O. Savitsky, E. A. Kravtsov, D. V. Blagodatkov, M. V. Ryabukhina, M. A. Milyaev, V. V. Ustinov, E. M. Pashaev, I. A. Subbotin, and G. V. Prutskov, Interlayer coupling in $\mathrm{Fe} / \mathrm{Cr} / \mathrm{Gd}$ multilayer structures, J. Exp. Theor. 120, 1041 (2015).

[30] A. B. Drovosekov, M. V. Ryabukhina, D. I. Kholin, N. M. Kreines, E. A. Manuilovich, A. O. Savitsky, E. A. Kravtsov, V. V. Proglyado, V. V. Ustinov, T. Keller, Y. N. Khaydukov, Y. Choi, and D. Haskel, Effect of cr spacer on structural and magnetic properties of Fe/Gd multilayers, J. Exp. Theor. 127, 742 (2018).

[31] K. Takanashi, H. Kurokawa, and H. Fujimori, A novel hysteresis loop and indirect exchange coupling in $\mathrm{Co} / \mathrm{Pt} / \mathrm{Gd} / \mathrm{Pt}$ multilayer films, Appl. Phys. Lett. 63, 1585 (1993).

[32] D. N. Merenkov, A. B. Chizhik, S. L. Gnatchenko, M. Baran, R. Szymczak, V. O. Vas'kovskiy, and A. V. Svalov, H-t phase diagram of a multilayered $\mathrm{Gd} / \mathrm{Si} / \mathrm{Co}$ film with ferrimagnetic ordering of the layers, Low Temp. Phys. 27, 137 (2001).
[33] S. G. E. te Velthuis, J. S. Jiang, S. D. Bader, and G. P. Felcher, Spin Flop Transition in a Finite Antiferromagnetic Superlattice: Evolution of the Magnetic Structure, Phys. Rev. Lett. 89, 127203 (2002).

[34] V. Lauter-Pasyuk, H. J. Lauter, B. P. Toperverg, L. Romashev, and V. Ustinov, Transverse and Lateral Structure of the SpinFlop Phase in $\mathrm{Fe} / \mathrm{Cr}$ Antiferromagnetic Superlattices, Phys. Rev. Lett. 89, 167203 (2002).

[35] D. L. Nagy, L. Bottyán, B. Croonenborghs, L. Deák, B. Degroote, J. Dekoster, H. J. Lauter, V. Lauter-Pasyuk, O. Leupold, M. Major, J. Meersschaut, O. Nikonov, A. Petrenko, R. Rüffer, H. Spiering, and E. Szilágyi, Coarsening of Antiferromagnetic Domains in Multilayers: The Key Role of Magnetocrystalline Anisotropy, Phys. Rev. Lett. 88, 157202 (2002).

[36] N. O. Antropov, Y. N. Khaydukov, E. A. Kravtsov, V. V. Makarova, M. V. Progliado, and V. V. Ustinov, Transition in a magnetic non-collinear spin-flop state in a $\mathrm{Fe} / \mathrm{Pd} / \mathrm{Gd} / \mathrm{Pd}$ superlattice, JETP Lett. 109, 406 (2019).

[37] E. Pashaev, A. Vasiliev, I. Subbotin, G. Prutskov, Y. M. Chesnokov, M. Kovalchuk, N. Antropov, E. Kravtsov, V. Proglyado, and V. Ustinov, Analysis of structural features of periodic $\mathrm{Fe} / \mathrm{Pd} / \mathrm{Gd} / \mathrm{Pd}$ multilayered systems, Crystallography Reports 65, 985 (2020).

[38] A. R. Stokes and A. J. C. Wilson, The diffraction of X-rays by distorted crystal aggregates, Proc. Phys. Soc. 56, 174 (1944).

[39] A. Solignac, R. Guerrero, P. Gogol, T. Maroutian, F. Ott, L. Largeau, P. Lecoeur, and M. Pannetier-Lecoeur, Dual Antiferromagnetic Coupling at $\mathrm{La}_{0.67} \mathrm{Sr}_{0.33} \mathrm{MnO}_{3} / \mathrm{SrRuO}_{3}$ Interfaces, Phys. Rev. Lett. 109, 027201 (2012).

[40] G. Suciu, J. Toussaint, and J. Voiron, 4f-3d exchange coupling in $\mathrm{gd} / \mathrm{x} / \mathrm{co}(\mathrm{x}=\mathrm{pt}, \mathrm{cr})$ multilayers, J. Magn. Magn. Mater. 240, 229 (2002).

[41] Y. N. Khaydukov, A. S. Vasenko, E. A. Kravtsov, V. V. Progliado, V. D. Zhaketov, A. Csik, Y. V. Nikitenko, A. V. Petrenko, T. Keller, A. A. Golubov, M. Y. Kupriyanov, V. V. Ustinov, V. L. Aksenov, and B. Keimer, Magnetic and superconducting phase diagram of $\mathrm{Nb} / \mathrm{Gd} / \mathrm{Nb}$ trilayers, Phys. Rev. B 97, 144511 (2018).

[42] Y. N. Khaydukov, E. A. Kravtsov, V. D. Zhaketov, V. V. Progliado, G. Kim, Y. V. Nikitenko, T. Keller, V. V. Ustinov, V. L. Aksenov, and B. Keimer, Magnetic proximity effect in $\mathrm{Nb} / \mathrm{Gd}$ superlattices seen by neutron reflectometry, Phys. Rev. B 99, 140503(R) (2019).

[43] B. Hjörvarsson, J. A. Dura, P. Isberg, T. Watanabe, T. J. Udovic, G. Andersson, and C. F. Majkrzak, Reversible Tuning of the Magnetic Exchange Coupling in Fe/V (001) Superlattices using Hydrogen, Phys. Rev. Lett. 79, 901 (1997).

[44] V. Leiner, K. Westerholt, B. Hjörvarsson, and H. Zabel, Tunability of the interlayer exchange coupling, J. Phys. D: Appl. Phys 35, 2377 (2002).

[45] A. Schreyer, K. Bröhl, J. F. Ankner, C. F. Majkrzak, T. Zeidler, P. Bödeker, N. Metoki, and H. Zabel, Oscillatory exchange coupling in $\mathrm{Co} / \mathrm{Cu}(111)$ superlattices, Phys. Rev. B 47, 15334 (1993).

[46] M. Hecker, S. Valencia, P. M. Oppeneer, H.-C. Mertins, and C. M. Schneider, Polarized soft-x-ray reflection spectroscopy of giant magnetoresistive $\mathrm{Co} / \mathrm{Cu}$ multilayers, Phys. Rev. B 72, 054437 (2005). 